\title{
Persistence of perfluoroalkylated substances in closed bottle tests with municipal sewage sludge
}

\author{
Monica Sáez • Pim de Voogt • John R. Parsons
}

Received: 14 May 2008 / Accepted: 27 May 2008 / Published online: 2 July 2008

(C) The Author(s) 2008

\begin{abstract}
Background, aim, and scope Perfluoroalkylated substances (PFAS) are chemicals with completely fluorinated alkyl chains. The specific properties of the $\mathrm{F}-\mathrm{C}$ bond give PFAS a high stability and make them very useful in a wide range of applications. PFAS also pose a potential risk to the environment and humans because they have been recently characterized as persistent, bioaccumulative, and toxic. The objective of this work is to study the bacterial degradation of PFAS under aerobic and anaerobic conditions in municipal sewage sludge as a contribution toward understanding their environmental fate and behavior.

Materials and methods Bacterial communities from sewage sludge were exposed to a mixture of PFAS under aerobic or anaerobic conditions. Individual PFAS concentrations were determined in the experiment media at different exposure
\end{abstract}

Responsible editor: Henner Hollert

M. Sáez • P. de Voogt •J. R. Parsons $(\bowtie)$

Department of Earth Surface Processes and Materials, IBED,

University of Amsterdam,

Nieuwe Achtergracht 166,

1018WV Amsterdam, The Netherlands

e-mail: jparsons@science.uva.nl

P. de Voogt

KIWA Water Research,

P.O. Box 1072, 3430 BB Nieuwegein, The Netherlands

Present address:

M. Sáez

Department of Instrumental Analysis and Environmental

Chemistry, Institute of Organic Chemistry,

CSIC, C/ Juan de la Cierva 3,

28006 Madrid, Spain times using liquid chromatography-mass spectrometry analysis after extraction with solid-phase extraction.

Results The PFAS analyses of samples of sludge showed repeatable replicate results, allowing a reliable quantification of the different groups of PFAS analyzed. No conclusive evidence for PFAS degradation was observed under the experimental conditions tested in this work. Reduction in concentrations, however, was observed for some PFAS in sludge under aerobic conditions.

Discussion The largest concentration decrease occurred for the fluorotelomer alcohols (FTOHs), especially for the 8:2 FTOH, which have been described as biodegradable in the literature. However, this concentration decrease could be due to different causes: sorption to glass, septa, or matrix components, as well as bacterial activity. Therefore, it is not certain that biodegradation occurred.

Conclusions PFAS are very recalcitrant chemicals, especially when fully fluorinated. Although some decreases in concentration have been observed for some PFAS, such as the FTOHs, there is no conclusive evidence for biodegradation. It can be concluded that the PFAS tested in these experiments are non-biodegradable under these experimental conditions. Recommendations and perspectives Since the presence of PFAS is ubiquitous in the environment and they can be toxic, more research is needed in this field to elucidate which PFAS are susceptible to biodegradation, the conditions required for biodegradation, and the possible routes followed. A possible inhibitory effect of PFAS on bacteria, the threshold concentrations, and conditions of inhibition should also be investigated.

Keywords Aerobic biodegradation .

Anaerobic biodegradation - Fluorotelomer alcohols .

FTOHs · Perfluoroalkylated substances · PFAS · Sludge 


\section{Background, aim, and scope}

Perfluoroalkylated substances (PFAS) are a group of chemicals, including oligomers and polymers, with unique properties due to their highly fluorinated structure. These properties make them suitable for a broad range of applications including surface treatment (oil-, grease-, and water-resistant coatings on paper and textile products) and performance chemicals (fire-fighting foams, industrial surfactants, acid mist suppression, insecticides, etc.; USEPA 2002; Hekster et al. 2003). These industrial and consumer uses have increased enormously over the last decades, and hence, the release of PFAS to the environment has also increased. A total of 3,200-7,300 tonnes of total PFAS have been estimated to have been globally discharged, both directly and indirectly (Prevedouros et al. 2006).

PFAS, mainly perfluorooctane sulfonate (PFOS) and perfluooctanoic acid (PFOA), have been detected in the abiotic environment (Berger et al. 2004; Yamashita et al. 2005; Skutlarek et al. 2006) and in biota (fish, birds, and mammals; Hansen et al. 2001) in a large part of the world, including such remote areas as the Arctic (Giesy and Kannan 2001; Tomy et al. 2004; Smithwick et al. 2005). Their transport to remote areas is unexpected because PFOS, PFOA, and related substances are much more watersoluble and less volatile than the typical persistent organic pollutants that undergo long-range transport. Recent studies suggest that fluorotelomer alcohols $(\mathrm{FTOH})$, which are volatile and can be more easily transported to remote locations, can be degraded to PFOA in the environment and hence may explain the discovery of PFOA in remote locations (Ellis et al. 2004, 2005; Berti et al. 2005). Another hypothesis is that direct long-range transport of PFOS and PFOA may occur via ocean currents (Taniyasu et al. 2004).

The widespread presence of PFAS in wildlife, their bioaccumulation, potential toxicity, and adverse health effects makes the study of their environmental fate a major concern. The extreme strength of the $\mathrm{C}-\mathrm{F}$ bond that makes PFAS suitable for certain uses also makes them potentially significant environmental contaminants due to their persistence. Although they are not easily degraded, there are few studies showing that a primary biodegradation of some perfluorinated compounds (like N-EtFOSE) to PFOS and PFOA (3M 2000a) can be observed, but there is no evidence for further degradation (3M 2000b, c, d, 2001).

It appears that aerobic biodegradation of polyfluorinated chemicals occurs in the non-fluorinated part of the molecule, without loss of fluorine (Remde and Debus 1996), and only takes place for non-perfluorinated compounds. Defluorination, however, has been reported for some fluorinated compounds containing carbon-hydrogen bonds, such as trifluoroethane sulfonate, 6:2 FTOH (Key et al. 1998), and recently, also for 8:2 FTOH (Dinglasan et al. 2004; Wang et al. 2005a, b). These last studies describe the aerobic degradation of 8:2 FTOH $\left(\mathrm{C}_{8} \mathrm{~F}_{17} \mathrm{CH}_{2} \mathrm{CH}_{2} \mathrm{OH}\right.$, the most commercially important FTOH) in sludge (Dinglasan et al. 2004; Wang et al. 2005a) and sediment (Wang et al. $2005 b$ ), as well as the possible degradation routes. Recently, biodegradation of FTOH, $N$-EtFOSAA, and other PFAS was proposed to contribute to the levels of PFOA and PFOS in waste water treatment plant sludge, effluents, and sediment (Higgins et al. 2005; Sinclair and Kannan 2006).

The objective of this paper is to contribute to a better understanding of the persistence of PFAS in the environment. For that purpose, biodegradation experiments of PFAS under aerobic and anaerobic conditions have been performed using bacterial consortia from municipal sewage sludge. Experiments have been performed with mixtures of PFAS since microorganisms in the environment are always exposed to mixtures of these compounds.

\section{Materials and methods}

\subsection{Chemicals}

High-performance liquid chromatography (HPLC) grade methanol $(\mathrm{MeOH})$ was purchased from Brunschwig Chemie, Amsterdam, The Netherlands. Deionized water was used throughout the experiments $(16.2 \mathrm{M} \Omega / \mathrm{cm})$. All chemicals were at least reagent grade. The biodegradation experiments were performed using mixtures of PFAS, which are shown in Table 1 together with their suppliers. All PFAS had purity higher than $96 \%$, and all concentrations reported in this paper were corrected for the purity. The $\mathrm{C}_{18}$ Sep-Pak cartridges were supplied by Waters Chromatography BV, Etten-Leur, The Netherlands, and the Acrodisc Syringe Filters with $0.2 \mu \mathrm{m}$ GHP Membrane were purchased from VWR International BV, Amsterdam, The Netherlands. The HPLC separation was performed with an Eclipse XDB C18 column of $150 \times 2.1 \mathrm{~mm}$ internal diameter and $3.5 \mu \mathrm{m}$ particle size, purchased from Agilent Technologies. Mass-labeled ${ }^{13} \mathrm{C}_{2}$-PFOA was used as internal standard.

\subsection{Experimental conditions}

The biodegradation experiments were based on the OECD guideline 301D (closed bottle test, OECD 1992) with slight modifications. Several batches of experiments were performed (Table 2) depending on the conditions (aerobic or anaerobic) and the mixture of PFAS tested. In all the experiments, bacteria were exposed to a mixture of PFAS with an initial nominal concentration of about 4 and $2 \mathrm{mg} /$ 1 for mixtures 1 and 2, respectively. Mixture 1 consists of 
Table 1 The two mixtures of PFAS used in the biodegradation experiments

\begin{tabular}{|c|c|c|c|c|}
\hline Mixture & PFAS & Structure & Abbreviation & Supplier \\
\hline \multirow[t]{4}{*}{1} & Undecafluorohexanoic acid & $\mathrm{C}_{5} \mathrm{~F}_{11} \mathrm{COOH}$ & PFHxA & $\mathrm{ABCR}$ \\
\hline & Pentadecafluorooctanoic acid & $\mathrm{C}_{7} \mathrm{~F}_{15} \mathrm{COOH}$ & PFOA & Acros \\
\hline & Heptadecafluorononanoic acid & $\mathrm{C}_{8} \mathrm{~F}_{17} \mathrm{COOH}$ & PFNA & Aldrich \\
\hline & Heptadecafluorooctanesulfonic acid (K salt) & $\mathrm{C}_{8} \mathrm{~F}_{17} \mathrm{SO}_{3} \mathrm{~K}$ & PFOS & Fluka \\
\hline \multirow[t]{6}{*}{2} & Nonafluorobutanesulfonic acid & $\mathrm{C}_{4} \mathrm{~F}_{9} \mathrm{SO}_{3} \mathrm{H}$ & PFBS & Apollo Sc. \\
\hline & Perfluoroundecanoic acid & $\mathrm{C}_{10} \mathrm{~F}_{21} \mathrm{COOH}$ & PFUnA & Apollo Sc. \\
\hline & Perfluoroctane sulfonamide & $\mathrm{C}_{8} \mathrm{~F}_{17} \mathrm{SO}_{2} \mathrm{NH}_{2}$ & PFOSA & $\mathrm{ABCR}$ \\
\hline & 1,1,2,2 H-perfluorooctane sulfonic acid & $\mathrm{C}_{6} \mathrm{~F}_{13} \mathrm{C}_{2} \mathrm{H}_{4} \mathrm{SO}_{3} \mathrm{H}$ & $6: 2$ FTS & Interchim \\
\hline & 1,1,2,2 H-perfluorooctanol & $\mathrm{C}_{6} \mathrm{~F}_{13} \mathrm{C}_{2} \mathrm{H}_{4} \mathrm{OH}$ & $6: 2 \mathrm{FTOH}$ & $\mathrm{ABCR}$ \\
\hline & 1,1,2,2 H-perfluorodecanol & $\mathrm{C}_{8} \mathrm{~F}_{15} \mathrm{C}_{2} \mathrm{H}_{4} \mathrm{OH}$ & $8: 2 \mathrm{FTOH}$ & DuPont \\
\hline IS & Pentadecafluorooctanoic acid & $\mathrm{C}_{7} \mathrm{~F}_{15} \mathrm{COOH}$ & ${ }^{13} \mathrm{C}-\mathrm{PFOA}$ & DuPont \\
\hline
\end{tabular}

PFHxA, PFOA, PFNA, and PFOS, whereas mixture 2 contains perfluorobutyl sulfonate (PFBS), perfluoroundecanoic acid (PFUnA), perfluorooctanesulfonamide (PFOSA), 6:2 fluorotelomer sulfonate (FTS), 6:2 FTOH, and 8:2 FTOH. All the PFAS tested are fully fluorinated, except the telomers 6:2 FTS, 6:2 FTOH, and 8:2 FTOH, which have two $\mathrm{CH}_{2}$ units.

Experimental bottles were run as independent duplicates with parallel sterile controls (bottles treated identically to experimental ones except for the autoclaving after inoculating). Each bottle contained $40 \mathrm{ml}$ mineral medium (12 $\mathrm{mM} \mathrm{KHPO}, 9 \mathrm{mM} \mathrm{Na} \mathrm{HPO}_{4}, 5.6 \mathrm{mM} \mathrm{NH} \mathrm{NH}_{4} \mathrm{Cl}$, $5.1 \mathrm{mM} \mathrm{NaCl}, 0.75 \mathrm{mM} \mathrm{CaCl}, 0.49 \mathrm{mM} \mathrm{MgCl}_{2}$, $9.5 \mathrm{mM} \mathrm{NaHCO}_{3}$, and $2 \mathrm{mM} \mathrm{Na}_{2} \mathrm{~S}$ ) and $10 \mathrm{ml}$ of inoculum (10 ml sludge supernatant). The reductant $\left(\mathrm{Na}_{2} \mathrm{~S}\right)$ was obviously absent from aerobic bottles. The bottles were kept under constant agitation and natural light period, although protected from UV radiation. The anaerobic experiments, kept in darkness, were performed under a nitrogen atmosphere in viton-sealed bottles, with resazurine as redox indicator.

Vitamins, trace elements, and a solution of lactate, acetic, and pyruvic acids, as extra carbon source, were also added. When most of the experiments had been running for 3-4 weeks (depending on the series), ethanol was added, since it has been shown to enhance the aerobic biodegradation of fluorotelomer alcohols (Wang et al. 2005b).

The activated sludge was taken from RWZI Westpoort, a waste water treatment plant in the West of the city of Amsterdam (The Netherlands), and transported to the laboratory where it was left for about $1-2 \mathrm{~h}$ to settle. Then, the supernatant was removed and kept with aeration until experiment bottles were filled (within a few hours).

\subsection{Analytical conditions}

\subsubsection{Sample extraction}

PFAS were extracted from supernatant of the sludge experiments (both aerobic and anaerobic) with $\mathrm{C}_{18} \mathrm{SPE}$ cartridges as described elsewhere (de Voogt et al, 2005). The cartridges were previously activated with $10 \mathrm{ml}$ of $\mathrm{MeOH}$ followed by $10 \mathrm{ml}$ of distilled water. The cartridges were eluted with $10 \mathrm{ml} \mathrm{MeOH}$, after which the volume was reduced under a gentle stream of $\mathrm{N}_{2}$ and filtered before analysis.

\subsubsection{Detection and quantification of PFAS}

PFAS analysis was performed using HPLC-electrospray ionization mass spectrometry (Thermoquest Navigator) under a flow of $0.25 \mathrm{ml} / \mathrm{min}$ of a mixture of $90 \%$ of $5 \mathrm{mM} \mathrm{CH}_{3} \mathrm{COONH}_{4}$ in water and $10 \%$ of $\mathrm{MeOH}$. At $12 \mathrm{~min}$, the $\mathrm{MeOH}$ was increased to $100 \%$, kept there for $2 \mathrm{~min}$, and returned to the initial condition in another $5 \mathrm{~min}$. The probe was kept at $220^{\circ} \mathrm{C}$ and $4.6 \mathrm{kV}$, while the entrance cone voltage was $-20 \mathrm{~V}$ (Sáez and de Voogt 2006). For identification and quantitative analysis, the masses $(\mathrm{m} / \mathrm{z})$ corresponding to the pseudo molecular ions $[\mathrm{M}-\mathrm{H}]^{-}$were selected (de Voogt and Sáez, 2006), except for the FTOHs,

Table 2 Experimental conditions of the different batches of the biodegradation experiments of PFAS

\begin{tabular}{llllll}
\hline Experiment code & Conditions & Medium & PFASs tested & Duration (months) & Sampling schedule (weeks) \\
\hline A 1 & Aerobic & Sludge & Mixture 1 & 3.5 & $0,1,2,3,7,15$ \\
A 2 & Aerobic & Sludge & Mixture 2 & 2 & $0,1,2,3,4,9$ \\
An 1 & Anaerobic & Sludge & Mixture 1 & 3.5 & $0,1,2,3,7,15$ \\
An 2 & Anaerobic & Sludge & Mixture 2 & 2 & $0,1,2,3,4,9$ \\
\hline
\end{tabular}


which were detected as the acetate adduct ions $[\mathrm{M}-\mathrm{H}$ $\left.+\mathrm{CH}_{3} \mathrm{COO}\right]^{-}$. The concentration of each PFAS was calculated using a five-point calibration curve determined with external standards. Concentrations were corrected for extraction and injection losses by the addition of ${ }^{13} \mathrm{C}_{2}$ PFOA before extraction. The average recovery of ${ }^{13} \mathrm{C}_{2}$ PFOA was $70.5 \pm 20.6 \%$. As the mass-labeled ${ }^{13} \mathrm{C}_{2}$-PFOA was not yet available during the first two experiments performed (A1, An1), 6:2 FTS was used as an internal standard in these experiments.

\section{Results}

The simultaneous analysis of different groups of PFAS presents several difficulties, such as extraction from complex matrices, lack of proper and well-characterized standards, and lack of robust analytical methods. The quality assurance in this study was realized through recovery tests and independent replicate analyses. Recoveries in general were satisfactory (greater than $60 \%$ ), and the replicates showed good repeatability.

\subsection{Aerobic experiments}

The time trends of the PFAS during the biodegradation experiments performed with sludge under aerobic conditions is shown in Fig. 1. No significant decrease is observed for any of the PFAS tested (with the exception of PFHxA, 6:2 FTOH, and 8:2 FTOH) over a period of up to either 9 or
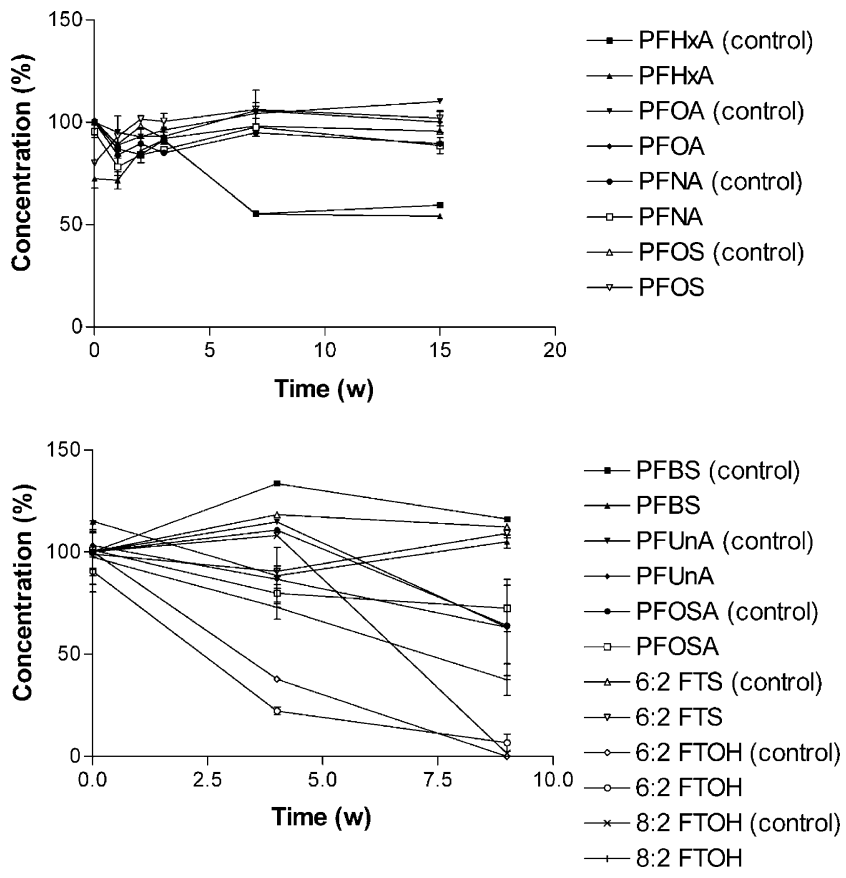

Fig. 1 Trends in the concentrations of PFAS (as percentage of the initial concentration) in sludge under aerobic conditions
15 weeks. The concentrations of PFHxA, 6:2 FTOH, and 8:2 FTOH also decreased in the controls during the experiment. The decrease is thus not conclusive evidence for biodegradation, since the decrease in concentration could be due to processes other than microbial metabolism. It is, however, also possible that incomplete sterilization of the controls could be responsible for the removal of these compounds in the controls.

\subsection{Anaerobic experiments}

Results of the experiments performed with sludge under anaerobic conditions are presented in Fig. 2, showing again a good reproducibility of the data. None of the tested compounds showed a significant decrease in the concentrations under these conditions during the course of the experiments ( 9 or 15 weeks, depending on the batch). Interestingly, there was no decrease in the concentrations of PFHxA, 6:2 FTOH, and 8:2 FTOH in these incubations, suggesting that the removal of these compounds in the aerobic experiments could indeed be due to biodegradation. The lack of biodegradation observed under anaerobic conditions is consistent with the fact that anaerobic biodegradation is a slower process than aerobic biodegradation.

\section{Discussion}

The simultaneous analysis of different groups of PFAS is complicated by the different properties of the FTOHs
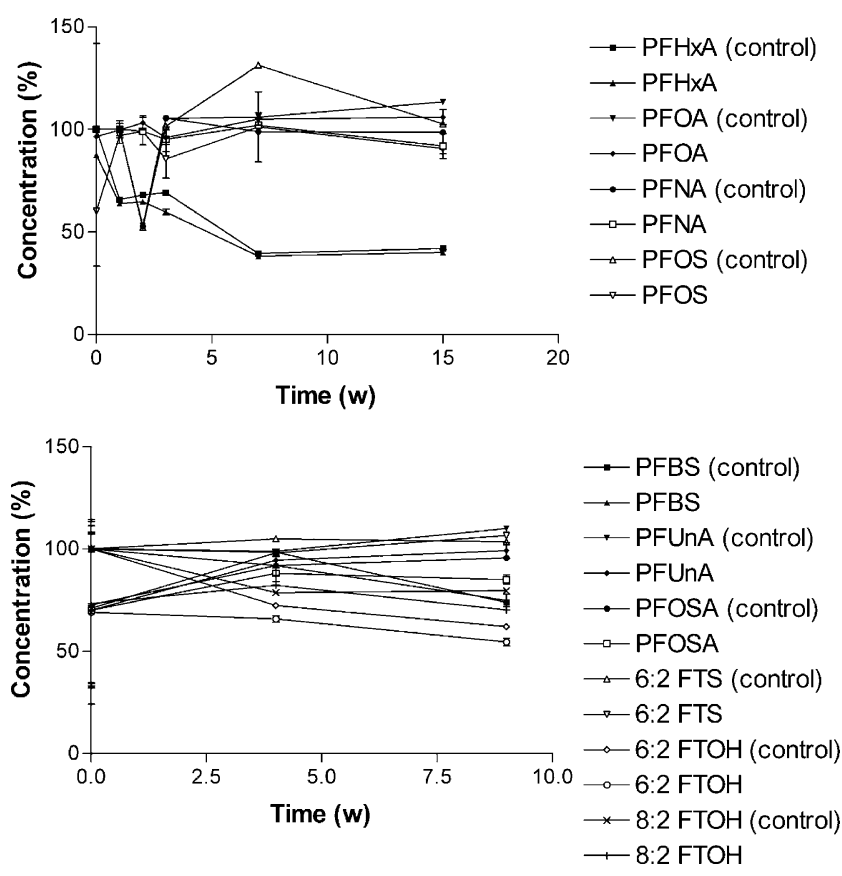

Fig. 2 Trends of the concentrations of PFAS (as percentage of the initial concentration) under anaerobic conditions 
(lower water solubility and higher volatility) compared with the perfluorocarboxylic acids and sulfonates, resulting in lower recoveries and higher detection limits, and therefore hindering the performance of the biodegradation experiments. The correction of the concentration of the tested PFAS with ${ }^{13} \mathrm{C}-\mathrm{PFOA}$ (internal standard) may lead to higher errors for those PFAS whose properties differ from those of PFOA. When the experiments were performed, this was the only labeled standard available, but this problem is nowadays being solved by the increasing number of masslabeled standards available. However, quality assurance measures gave satisfactory results.

There is no conclusive evidence for biodegradation of PFAS in sludge under aerobic conditions. Although decreases in PFHxA, 6:2 FTOH, and 8:2 FTOH concentrations were observed, their concentrations in the control bottles also decreased, and it is therefore not possible to confirm that they were indeed due to biodegradation. The concentration decrease could also be due to a nonbiological degradation process, losses not due to degradation or even to incomplete sterilization of the control bottles. Aerobic biodegradation of 8:2 FTOH has been reported in sludge by Dinglasan et al. (2004) and by Wang et al. $(2005 \mathrm{a}, \mathrm{b})$, but this could not be confirmed in the present experiments. No evidence for degradation of any of the PFAS was observed under anaerobic conditions. This result is consistent with the lack of anaerobic biodegradation of PFAS reported elsewhere in the literature.

Based on the results presented in this paper, it can therefore be concluded that the PFAS tested in these experiments are non-biodegradable under the experimental conditions used in this study, despite using municipal sewage sludge, which presumably has a history of exposure to PFAS. Similar experiments with sediment contaminated with PFAS also showed no evidence for biodegradation of any of the PFAS tested (data not shown).

\section{Conclusions and recommendations}

It is reasonable to assume that the lack of degradation of PFAS observed in these experiments is probably caused by the stability of the $\mathrm{C}-\mathrm{F}$ bond, although there are examples of microbially catalyzed defluorination reactions (Parsons et al. 2008). As is the case with reductive dechlorination or debromination, reductive defluorination is energetically favorable and, under anaerobic conditions, releases more energy than that available as a respiratory process from sulfate reduction or methanogenesis. Several species of anaerobic microorganisms are known to utilize reductive dechlorination as a respiratory process. We should therefore consider the possibility that microorganisms will eventually adapt to utilize defluorination as a source of energy. Hence, the situation for PFAS may be comparable to that of chlorinated organic compounds several decades ago. For many years, organochlorine compounds were considered to be recalcitrant, whereas today reductive chlorination reactions of many organochlorines, including polychlorinated biphenyls and dioxins, are regularly observed in anaerobic environments. Further studies should, however, be performed to investigate whether longer exposure times or higher exposure concentrations could lead to the development of degradation capability in microbial populations in sludges and sediments.

Acknowledgment This research work was financially supported by the European Union, Project PERFORCE (NEST-508967); by the Ministerio de Educación y Ciencia (Spanish Ministry for Education and Science); and DuPont Chemical Solutions Enterprise, Wilmington, Delaware, USA. The authors would like to thank the staff of the RWZI Westpoort for their assistance in the sludge sampling.

Open Access This article is distributed under the terms of the Creative Commons Attribution Noncommercial License which permits any noncommercial use, distribution, and reproduction in any medium, provided the original author(s) and source are credited.

\section{References}

3M (2000a) The aerobic biodegradation of N-EtFOSE alcohol by the microbial activity present in municipal wastewater treatment sludge.. Pace Analytical Services, Minneapolis, MN

3M (2000b) Microbial metabolism (biodegradation). Studies of perfluorooctane sulfonate (PFOS). II-aerobic soil degradation. Springborn Laboratories, Wareham, MA

3M (2000c) Microbial metabolism (biodegradation). Studies of perfluorooctane sulfonate (PFOS). IV-pure culture study. Springborn Laboratories, Wareham, MA

3M (2000d) Microbial metabolism (biodegradation). Studies of perfluorooctane sulfonate (PFOS). III-anaerobic sludge biodegradation. Springborn Laboratories, Wareham, MA

3M (2001) The 18-day aerobic biodegradation study of perfluorooctanesulfonyl-based chemistries. Pace Analytical Services, Minneapolis, $\mathrm{MN}$

Berger U, Järnberg U, Kallenborn R (2004) Perfluorinated alkylated substances (PFAS) in the European Nordic environment. Organohalog Compd 66:4046-4052

Berti WR, Szostek B, Buck RC, Schaefer E, Van Hoven RL, Wang N, Gannon JT (2005) Biodegradation studies of fluorotelomer-based polymers to assess their potential to contribute to perfluorinated carboxylic acids in the environment. Proceedings FluorosInternational Symposium on Fluorinated Alkyl Organics in the Environment in Toronto

de Voogt P, Sáez M (2006) Analytical chemistry of perfluoroalkylated substances. Trends Anal Chem 25:326-342

de Voogt P, Sáez M, van Roon A (2005) Perfluorinated chemicals in sediments, particulate matter, soil and water samples from The Netherlands. Organohalog Compd 67:790-793

Dinglasan MJ, Ye Y, Edwards EA, Mabury SA (2004) Fluorotelomer alcohol biodegradation yields poly- and perfluorinated acids. Environ Sci Technol 38:2857-2864 
Ellis DA, Martin JW, De Silva AO, Mabury SA, Hurley MD, Andersen MPS, Wallington TJ (2004) Degradation of fluorotelomer alcohols: a likely atmospheric source of perfluorinated carboxylic acids. Environ Sci Technol 38:3316-3321

Ellis DA, Martin JW, Mabury SA, Hurley MD, Sulbaek-Andersen MP, Wallington TJ (2005) The degradation of fluorotelomer alcohols in the troposphere. Proceedings Fluoros-International Symposium on Fluorinated Alkyl Organics in the Environment in Toronto

Giesy JP, Kannan K (2001) Global distribution of perfluorooctane sulfonate in wildlife. Environ Sci Technol 35:1339-1342

Hansen KJ, Clemen LA, Ellefson ME, Johnson HO (2001) Compoundspecific quantitative characterization of organic fluorochemicals in biological matrices. Environ Sci Technol 35:766-770

Hekster FM, Laane RW, de Voogt P (2003) Environmental and toxicity effects of perfluoroalkylated substances. Rev Environ Contam Toxicol 179:99-121

Higgins CP, Field JA, Criddle CS, Luthy RG (2005) Quantitative determination of perfluorochemicals in sediments and domestic sludge. Environ Sci Technol 39:3946-3956

Key BD, Howell RD, Criddle CS (1998) Defluorination of organofluorine sulfur compounds by Pseudomonas Sp strain D2. Environ Sci Technol 32:2283-2287

OECD (1992) Ready biodegradability. OECD guidelines for testing chemicals - 301 D close bottle test. OECD, Paris

Parsons JR, Sáez M, Dolfing J, de Voogt P (2008) Biodegradation of perfluorinated compounds. Rev Environ Contam Toxicol 196 (in press)

Prevedouros K, Cousins IT, Buck RC, Korzeniowski SH (2006) Sources, fate and transport of perfluorocarboxylates. Environ Sci Technol 40:32-44

Remde A, Debus R (1996) Biodegradability of fluorinated surfactants under aerobic and anaerobic conditions. Chemosphere 32:1563-1574

Sáez M, de Voogt P (2006) Optimization of the determination and quantification of perfluorinated compounds by LC-ESI-MS. 2nd International Workshop on LC-MS/MS for Screening and Trace Level Quantitation in Environmental and Food Samples, Barcelona

Sinclair E, Kannan K (2006) Mass loading and fate of perfluoroalkyl surfactants in wastewater treatment plants. Environ Sci Technol 40:1408-1414

Skutlarek D, Exner M, Färber H (2006) Perfluorinated surfactants in surface and drinking water. Environ Sci Pollut Res 5:299-307

Smithwick M, Mabury SA, Solomon KR, Sonne C, Martin JW, Born EW, Dietz R, Derocher AE, Letcher RJ, Evans TJ, Gabrielsen GW, Nagy J, Stirling I, Taylor MK, Muir DCG (2005) Circumpolar study of perfluoroalkyl contaminants in polar bears (Ursus maritimus). Environ Sci Technol 39:5517-5523

Taniyasu S, Yamashita N, Kannan K, Horii Y, Sinclair E, Petrick G, Gamo T (2004) Perfluorinated carboxylates and sulfonates in open ocean waters of the Pacific and Atlantic Oceans. Organohalog Compd 66:4035-4040

Tomy GT, Budakowski WR, Halldorson T, Helm PA, Stern GA, Friesen K, Pepper K, Tittlemier SA, Fisk AT (2004) Fluorinated organic compounds in an Eastern arctic marine food web. Environ Sci Technol 38:6475-6481

USEPA (2002) Draft hazard assessment of perfluorooctanoic acid and its salts. USEPA, Washington, DC

Wang N, Szostek B, Folsom PW, Sulecki LM, Capka V, Buck RC, Berti WR, Gannon JT (2005a) Aerobic biotransformation of C-14labeled 8-2 telomer B alcohol by activated sludge from a domestic sewage treatment plant. Environ Sci Technol 39:531-538

Wang N, Szostek B, Buck RC, Folsom PW, Sulecki LM, Capka V, Berti WR, Gannon JT (2005b) Fluorotelomer alcohol biodegradation-direct evidence that perfluorinated carbon chains breakdown. Environ Sci Technol 39:7516-528

Yamashita N, Kannan K, Taniyasu S, Horii Y, Petrick G, Gamo T (2005) A global survey of perfluorinated acids in oceans. Mar Pollut Bull 51:658-668 